\title{
Hábitos alimenticios del pez sable Trichiurus lepturus en el Golfo de Urabá, Caribe colombiano
}

\author{
Feeding habits of the Atlantic Cutlass fish Trichiurus \\ lepturus in the Urabá Gulf, Colombian Caribbean
}

\author{
Sara Arenas-Uribe ${ }^{()}$, Jenny Leal-Flórez ${ }^{\circledR}$, Alejandro Sandoval ${ }^{\circledR}$, Andres Felipe Pérez Villa ${ }^{\circledR}$ y \\ Andres Felipe Hernández-Morales ${ }^{(0)}$
}

\section{Resumen}

Se estudiaron los hábitos alimenticios del pez comercial Trichiurus lepturus, un mesodepredador con un papel importante en el flujo de energía en las redes tróficas costeras, con el objeto de contribuir al conocimiento de su ecología, herramienta para su conservación e insumo para el ordenamiento pesquero del Golfo de Urabá, en el noroeste de Colombia. Usando redes de enmalle se capturaron 320 individuos, de los que se obtuvieron 208 estómagos con contenido. El espectro alimenticio de la especie está integrado por 26 ítems, pertenecientes a tres grandes grupos taxonómicos: peces, crustáceos y moluscos. Entre estos, los engráulidos (Engraulidae) son el principal componente en todos los sitios de muestreo, sobresaliendo Centengraulis edentulus y varias especies del género Anchoa. Se encontró un traslape de presas entre los sitios, siendo evidente la importancia de las áreas de manglar para alimentación. Sin embargo, no hubo variación estacional ni diferencias entre sexos en la composición de la dieta, dado que los engráulidos, su principal presa, son uno de los recursos pesqueros más abundantes en el Caribe colombiano.

Palabras clave. Análisis de contenido estomacal. Dieta de peces. Ecología trófica. Ítems alimenticios.

\begin{abstract}
We studied the feeding habits of the commercial fish Trichiurus lepturus, a mesopredator with an important role in the energy flow in the coastal trophic webs, in order to contribute to the knowledge of its ecology, tool for its conservation, and an input for the management of fisheries in the Gulf of Urabá, northwestern Colombia. Using gillnets, 320 fish were captured, from which 208 stomachs containing food were extracted, and their contents separated and identified. The food spectrum of the species is integrated by 26 elements, belonging to three great taxonomic groups: fish, crustaceans and mollusks. Engraulid fishes are the main food component at all sampling sites, mosly Centengraulis edentulus and several Anchoa species. There was a trophic overlap among sites, and the importance of mangrove areas for feeding was evident. However, no seasonal or sex variation in diet composition was found, since Engraulids are one of the most abundant fish resources throughout the year in the Colombian Caribbean.
\end{abstract}

Keywords. Stomach content analysis. Fish diet. Trophic ecology. Food items. 


\section{Introducción}

La familia Trichiuridae comprende 10 géneros y 39 species de peces con talla grande, cuerpo alargado y lateralmente comprimido, aletas pélvicas reducidas o ausentes y dientes triangulares y agudos (Randall, 1967; Nelson, 2006; García, 2010). Son depredadores importantes con hábitos generalmente diurnos y altamente selectivos (Ros \& Pérez, 1978; Pardo et al., 2003).

Para el Caribe colombiano se han reportado cuatro especies de la familia Trichiuridae: Benthodesmus simonyi, B. tenius, T. lepturus y Lepidopus caudatus, entre las cuales T. lepturus destaca por ser la especie más abundante e importante en el comercio según Chakraborty et al. (2005). Este pez cosmopolita y pelágico, habita en aguas cálidas tropicales y subtropicales, moviéndose entre estuarios, manglares y mar abierto, de acuerdo a su ciclo de vida y demanda de alimento (Nakamura \& Parin, 1993; FAO, 2016; Bittar et al., 2012). En el golfo de Urabá, T. lepturus, conocido comúnmente como sable, es frecuente en el comercio de la zona, pese a tener un bajo valor comercial, y es usado además como carnada para la captura de otros recursos pesqueros (Datos sin publicar del proyecto LOPEGU; Leal-Flórez, J. et al., 2017).

Debido a la sobreexplotación de gran cantidad de pesquerías, los recursos que estas brindan disminuyen y se pone en peligro su supervivencia (Robles, 2014; Riveiro, 2015; FAO, 2016). Esta situación ha llevado a buscar enfoques diferentes para administrar adecuadamente los ecosistemas, por lo que es indispensable tener en cuenta la estructura de las comunidades de peces, la transferencia de energía y los cambios tróficos en el ecosistema (Morzaria et al., 2014; Hobday et al., 2015). Para ello es necesario estudiar los hábitos alimenticios de las especies que habitan un lugar (Pardo et al., 2003; Vaslet et al., 2015).

Este tipo de estudios permiten ampliar el conocimiento sobre la estructura y funcionamiento de una población y comprender mejor la biología y ecología de los recursos y el papel que juegan en los ecosistemas (Chiappa et al.,
1989; Bax, 1998; Pardo et al., 2003; Agudelo et al., 2011). Por esta razón se estudiaron los hábitos alimenticios del sable T. lepturus en el Golfo de Urabá en el Caribe colombiano, con el objeto de contribuir al conocimiento de su ecología, herramienta para su conservación e insumo para el ordenamiento pesquero de la zona.

Existen en el mundo varias investigaciones sobre interacciones biológicas, solapamiento de dieta y hábitos alimenticios del T. lepturus. En Asia, algunos de los estudios más destacados son sobre su estructura de tallas, reproducción, edad, crecimiento (Al-Nahdi et al., 2009) y sus hábitos alimenticios, encontrando tendencias hacia el consumo de camarones, calamares y peces (Chiou et al., 2006; Yan et al., 2011; Rohit et al., 2015). En África se también se encontró que sus principales alimentos son crustáceos, cefalópodos y peces (Wojciechowski, 1972), además de otra variedad de presas como huevos de peces, anfípodos y copépodos (Bakhoum, 2007).

En Suramérica se estudiaron los estómagos de larvas, juveniles y adultos, encontrando que se alimentan de larvas de copépodos, zooplancton y peces (Martins et al., 2005). Se comparó la dieta de T. lepturus con la de Pontoporia blainvillei, encontrando que ambas se solapan y los ítems más representativos son peces teleósteos de las familias Scianidae, Engraulidae y Clupeidae (Bittar \& Di Beneditto, 2009). Posteriormente, se investigó la alimentación hembras adultas a través de valores calóricos, hallando nuevamente preferencia por presas como peces y camarones (Bittar et al, 2012). Para el Caribe colombiano sólo se ha determinado que en el área de Cartagena la dieta del T. lepturus está conformada únicamente por peces, entre los cuales se destacan los mugílidos, engráulidos, carángidos, esfirénidos, sciaénidos, escómbridos y trichúridos (Pardo et al., 2003). 


\section{Materiales y métodos}

Área de estudio. El Golfo de Urabá está ubicado en la esquina suroccidental del Caribe colombiano, la zona más austral del mar Caribe (Figura 1), entre los $7^{\circ} 55^{\prime} \mathrm{N}$ y $8^{\circ} 40^{\prime} \mathrm{N}$ y los $76^{\circ} 53^{\prime} \mathrm{O}$ y $77^{\circ} 23^{\prime} \mathrm{O}$. Tiene cerca de $600 \mathrm{~km}$ de costa entre Arboletes y Cabo Tiburón. Abundan las zonas someras, no superando los $60 \mathrm{~m}$ de profundidad al norte y los $30 \mathrm{~m}$ al sur en Bahía Colombia (Chevillot et al., 1993; Bernal et al., 2005; Ortiz \& Blanco, 2012).

En el golfo existe un gradiente sur-norte de humedad, que determina una variación en el clima. La temperatura media superficial de la atmósfera registrada para la zona es de $26{ }^{\circ} \mathrm{C}$ a $28^{\circ} \mathrm{C}$ (Blanco-Libreros et al., 2015), aunque en época de verano (diciembre-marzo) se han encontrado temperaturas superiores a $40{ }^{\circ} \mathrm{C}$ y predominan los vientos alisios del norte y noreste, que confinan las aguas del río Atrato al interior del golfo. En invierno (agosto-noviembre) la temperatura desciende hasta $19^{\circ} \mathrm{C}$, se presentan vientos con direcciones variables, que contribuyen a que las aguas sean evacuadas, siendo esta la época con mayor salinidad (Chevillot et al., 1993; Ortiz \& Blanco, 2012). Las mareas del golfo son de tipo micromareal y no sobrepasan los $40 \mathrm{~cm}$ (Restrepo \& Correa, 2002).

La circulación del golfo, que es influenciada por los vientos y las mareas, pone en interacción las aguas del mar Caribe y del río Atrato, para dar origen a un estuario (Bernal et al., 2005), que presenta estratificación salina por la pluma superficial del río Atrato (Montoya \& Toro, 2006).

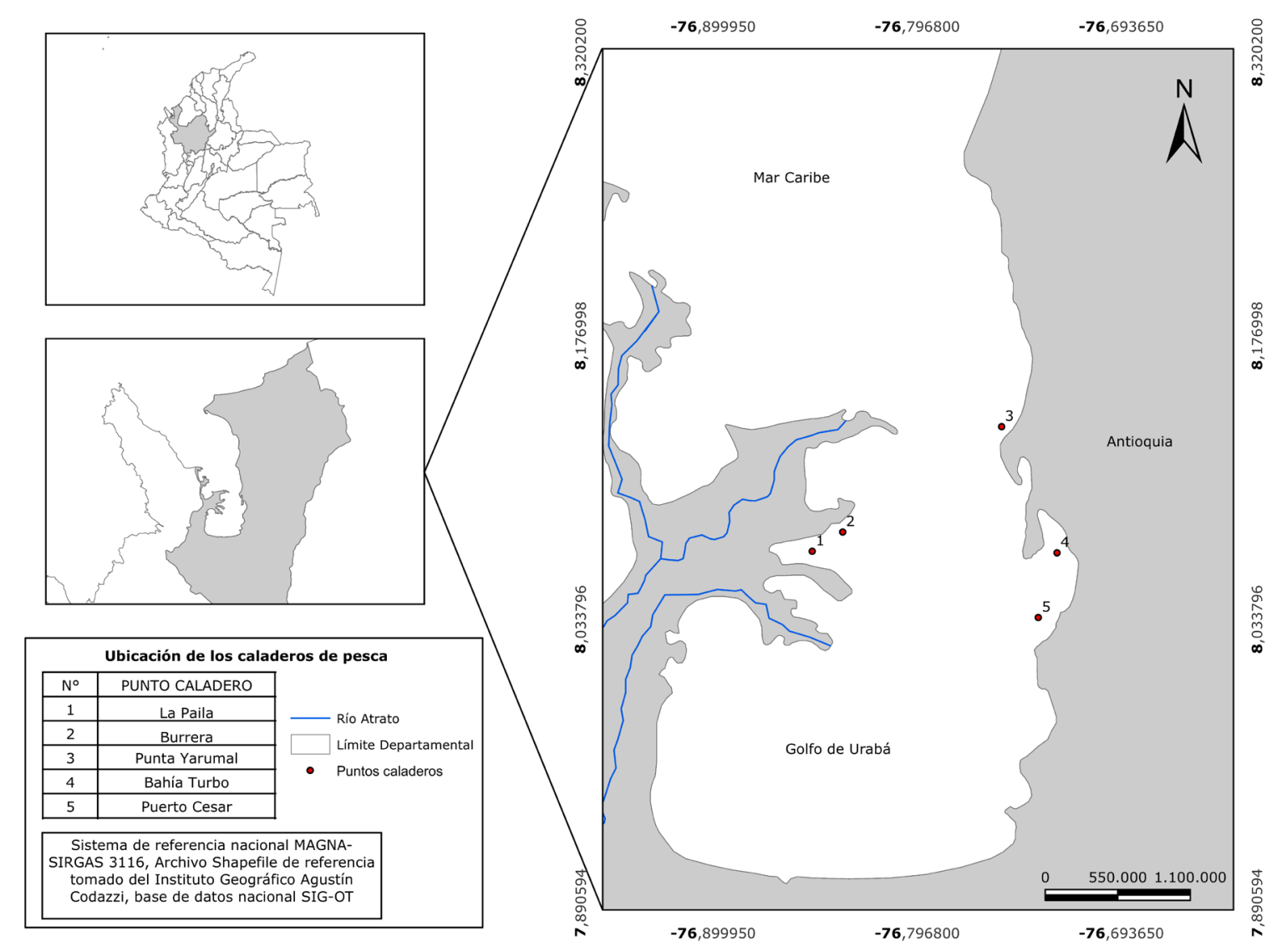

Figura 1. Puntos de muestreo de Trichiurus lepturus en el Golfo de Urabá, Caribe colombiano. El área en gris corresponde al departamento de Antioquia. 
Diseño del muestreo. Los ejemplares de T. lepturus fueron obtenidos entre agosto de 2015 y agosto de 2016, en cinco zonas de muestreo (Figura 1), definidas según la cercanía con las áreas de caladeros de pesca de mayor importancia en el golfo, determinadas por la Autoridad Nacional de Acuicultura y Pesca (AUNAP), que coinciden, en general, con áreas de desembocadura de ríos y zonas de bosque de manglar (Figura 1).

Se hicieron seis muestreos bimensuales, en los que se capturaron los especímenes empleando redes de enmalle monofilamento y multifilamento, con ojos que variaban entre dos y tres puntos. Adicionalmente, se obtuvieron ejemplares comprados a pescadores artesanales, que incluían la información espacial y temporal pertinente para su análisis.

Los individuos recolectados se sacrificaron usando una sobredosis de Eugenol en un balde con agua (Rubio \& Silveira, 2009). In situ, cada ejemplar fue inyectado con formol al $4 \%$ en la parte ventral para detener la actividad enzimática y procesos metabólicos estomacales (Borski \& Hodson, 2003). Posteriormente, fueron llevados a los laboratorios de la sede Ciencias del Mar de la Universidad de Antioquia, en Turbo, para ser pesados y medidos, utilizando una balanza My weigth i Balance 5500 con 0.01 mg de precisión y un ictiómetro artesanal de $1 \mathrm{~mm}$ de precisión. Un ejemplar de T. lepturus fue depositado en el Museo de Historia Natural Marina de Colombia (MHNMC) en el INVEMAR. Los estómagos fueron extraídos mediante un corte ventral desde el ano hacia la región pectoral (Moreno et al., 2009) y fijados en formol al $4 \%$ durante dos días y luego en alcohol al 40 \% (modificado de Marrero, 1994) para su posterior análisis.

Análisis de dietas.La representatividad del tamaño de muestra fue estimada mediante la propuesta de Ferry et al. (1997), calculando la media acumulada y la desviación estándar de un grupo de curvas de diversidad, generadas mediante el índice de ShannonWiener ' $\mathrm{H}$ ', con los datos de abundancia de las presas (Shannon-Wiener, 1948; Krebs, 1989). Los peces cuyo estómago tenía alimento fueron divididos en dos intervalos de talla con igual rango, así: talla pequeña
$(\mathrm{LT}=48.5-71.95 \mathrm{~cm})$ y talla grande $(\mathrm{LT}=71.96-95.4$ $\mathrm{cm})$. Los rangos no tienen en cuenta la información de talla media de madurez sexual, debido a que todos los individuos capturados superan la talla crítica $(46.3 \mathrm{~cm}$ en Froese \& Pauly, 2019).

Se calculó el índice de repleción (IR= Peso del contenido estomacal/Peso total del pez x 100), para estimar la condición de llenado de los estómagos (Hyslop, 1980), teniendo en cuenta la escala propuesta por Franco \& Bashirullah (1992): IR < 0.5, estómago semivacío; 0.5 $<$ IR < 1.0, estómago semilleno; IR > 1.0, estómago lleno. También se utilizó el coeficiente de vacuidad (V = número de estómagos vacíos/total del número de estómagos x 100), para determinar tasa de actividad alimentaria (Windell, 1971). Se calculó un indicador de gremio trófico, utilizando la relación: Longitud del intestino/Longitud total, para establecer si la especie es carnívora (relación <1), omnívora (relación entre 2 y 5 ) o herbívora (relación >5) (Kappor et al., 1976).

El contenido estomacal fue depositado en cajas de Petri y, con la ayuda de un estereoscopio Led trilocular Motic SMZ 168T, se separaron las presas contenidas por grupos taxonómicos o ítems alimentarios (crustáceos, moluscos, peces, restos de pez y material no identificable). Las presas encontradas en los contenidos estomacales se identificaron hasta el menor nivel taxonómico posible, utilizando las claves de Brusca \& Brusca (2005), Carpenter (2002) y Ruppert \& Barnes (1996) y Cervigón et al. (1992), siguiendo las técnicas estandarizadas para estudios de dietas de peces propuestas por Hyslop (1980) y Marrero (1994).

Se calculó el porcentaje de digestión de las presas en cada estómago, según la propuesta de GalvánMagaña et al. (1989): estado 1 (presas recientes): ítems completos; estado 2 (intermedio): presas en descomposición, pero con presencia de músculo u otros tejidos; estado 3 (semidigerido): presencia de esqueletos de peces o exoesqueletos de crustáceos; y estado 4 (digerido): presencia de partes duras, otolitos de peces y mandíbulas de cefalópodos. En organismos en los que se encontraban presas altamente digeridas, se emplearon otolitos para una identificación 
más precisa, contrastando con otolitos de presas ya identificadas con menor grado de digestión o de la colección de referencia construida para tal fin en el proyecto, usando claves de identificación como las de Jobling \& Breiby (1986), Volpedo \& Echeverria (1999), Popper \& Lu (2000), García et al. (2004), Martínez et al. (2007), Merchant et al. (2008) y Díaz (2010).

Para el análisis de su contribución a los hábitos alimenticios de los sables, cada presa fue registrada por conteo numérico (\% $\%$ número de una presa/ número de todas las presas x 100) (Hyslop, 1980; Bowen, 1996), porcentaje en peso $(\% \mathrm{P}=$ peso de una presa/peso de todas las presas x 100) (Hynes, 1950; Hyslop, 1980; Bowen, 1996) y frecuencia de aparición $\left(\% \mathrm{FA}=\mathrm{FA}\right.$ de una presa $/ \sum \mathrm{FA}$ de todas las presas $\mathrm{x}$ 100) (Hyslop, 1980). Posteriormente, para determinar la importancia de los ítems consumidos, se calculó el índice de importancia relativa $(\mathrm{IIR}=(\% \mathrm{~N}+\% \mathrm{P}) * \% \mathrm{FA})$ (Pinkas et al., 1970). Este índice presenta un rango de 0 a $100 \%$, donde valores de 0 a $10 \%$ representan grupos tróficos de importancia relativa baja u ocasionales, 10 a 40\% grupos de importancia relativa secundaria o frecuentes y valores superiores a $40 \%$, grupos de importancia relativa alta (Yáñez-Arancibia et al., 1985).

Se utilizó el índice de Morisita-Horn, para evidenciar el posible solapamiento trófico entre los sitios de captura. Los valores de este índice oscilan entre 0 y 1 , representando un traslape significativo cuando se alcanza un valor superior a 0.60 (Horn, 1966):

$$
\mathrm{C} \lambda=\frac{2 \sum_{\mathrm{i}=1}^{\mathrm{n}}\left(\mathrm{P}_{\mathrm{xi}} * \mathrm{P}_{\mathrm{yi}}\right)}{\left(\sum_{\mathrm{i}=1}^{\mathrm{n}} \mathrm{P}_{\mathrm{xi}^{2}}+\sum_{\mathrm{i}=1}^{\mathrm{n}} \mathrm{P}_{\mathrm{yi}^{2}}\right)}
$$

Donde:

$\mathrm{C} \lambda=$ Índice de Morisita-Horn de traslapo.

$\mathrm{n}=$ Número total de presas.
$\mathrm{P}_{\mathrm{xi}}=$ Proporción de la presa i del total de presas usadas por el sitio $x$.

$\mathrm{P}_{\mathrm{yi}}=$ Proporción de la presa i del total de las presas usadas por el sitio $\mathrm{y}$.

Se verificaron los supuestos de normalidad y homogeneidad de varianza y se aplicó la prueba $U$ de Mann-Whitney (Mann \& Whitney, 1947), con el fin de comprobar la existencia de diferencias significativas en la dieta entre longitudes, sexos y épocas. Estos análisis estadísticos se realizaron utilizando IBM SPSS Statistics 24.0, con significancia estadística igual o menor a 0.05 .

\section{Resultados}

Se recolectaron 320 individuos de T. lepturus, de os cuales el $84.5 \%$ fueron capturados en el día y el $15.5 \%$ en la noche. De los 320 estómagos, 208 tenían contenido. La curva de diversidad de presas de T. lepturus alcanzó la asíntota en 178 estómagos, confirmando el tamaño más que adecuado de la muestra para describir con precisión la composición de la dieta de la especie (Figura 2). El índice de vacuidad fue de 35 \% (112 estómagos vacíos). La condición de llenado para los estómagos con contenido estomacal, calculada con el índice de repleción, indicó que el 89.9 $\%$ se encontraron en la categoría lleno, el $4.8 \%$ en semilleno y el $5.2 \%$ en semivacíos.

El espectro alimentario estuvo integrado por 26 ítems (Tabla 1), pertenecientes en su orden a tres grandes grupos taxonómicos: peces, crustáceos y moluscos. Se logró identificar un ítem a nivel de clase, 12 familias, dentro de las cuales se identificaron 2 géneros y 13 ítems a nivel de especie. 


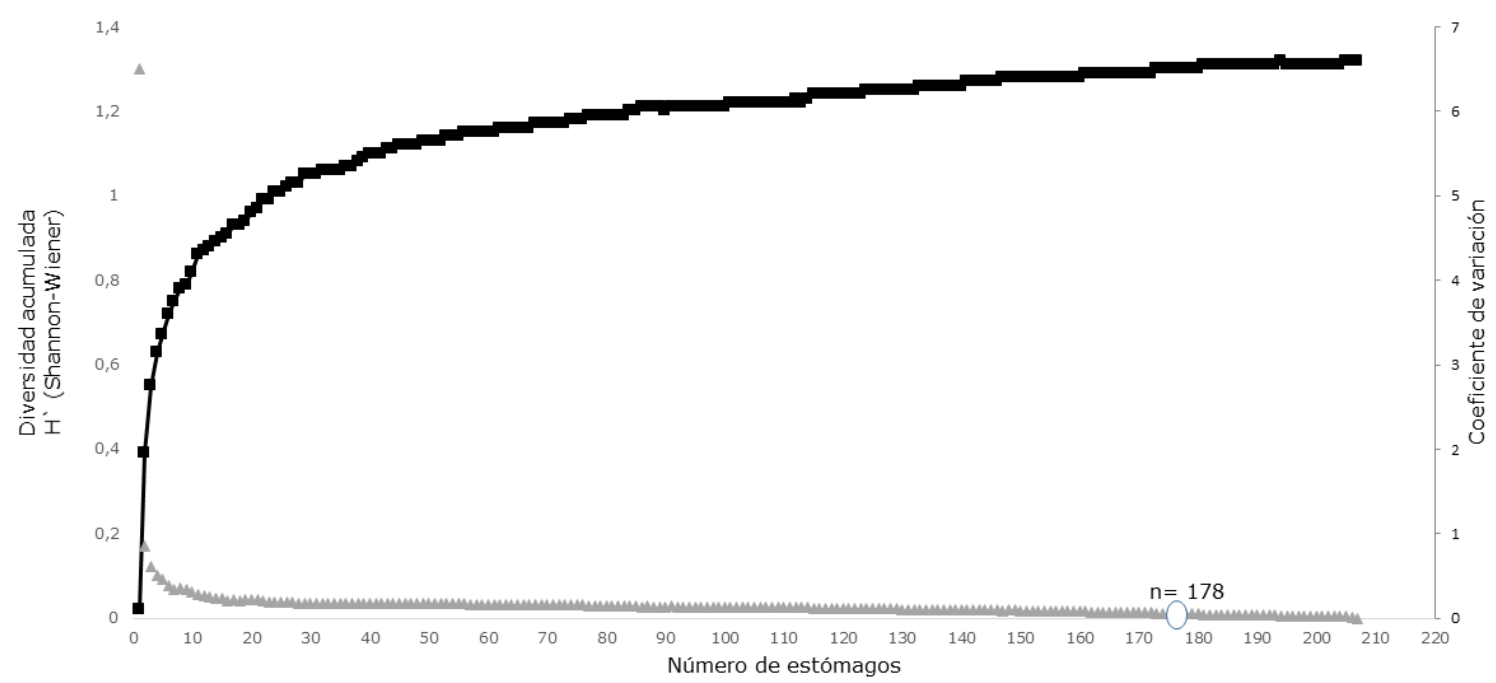

Figura 2. Curva de diversidad trófica (Shannon-Wiener, H’s) de Trichiurus lepturus capturados en el Golfo de Urabá, Caribe colombiano.

Tabla 1. Tabla 1. Porcentaje numérico $(\% \mathrm{~N})$, porcentaje en peso $(\% \mathrm{P})$, frecuencia de aparición $(\% \mathrm{FA})$ e índice de importancia relativa (\%IIR) para cada ítem de la dieta de Trichiurus lepturus en el Golfo de Urabá, Caribe colombiano. Los ítems más importantes están señalados con asterisco $\left(^{*}\right)$.

\begin{tabular}{|c|c|c|c|c|}
\hline Ítems Alimenticios & $\% \mathrm{~N}$ & $\% P$ & $\%$ FA & \%IIIR \\
\hline \multicolumn{5}{|l|}{ Crustáceos } \\
\hline Peneaidae & 0.279 & 0.003 & 0.481 & 0.002 \\
\hline Portunidae & 0.279 & 0.000 & 0.481 & 0.002 \\
\hline \multicolumn{5}{|l|}{ Moluscos } \\
\hline Cephalopoda & 0.279 & 0.003 & 0.481 & 0.002 \\
\hline \multicolumn{5}{|l|}{ Peces } \\
\hline \multicolumn{5}{|l|}{ Pristigasteridae } \\
\hline Pellona harroweri & 1.950 & 0.021 & 3.365 & 0.101 \\
\hline \multicolumn{5}{|l|}{ Engraulidae } \\
\hline Anchoa spinifer & 2.228 & 0.040 & 3.846 & 0.133 \\
\hline Anchoa filifera & 2.228 & 0.037 & 3.846 & 0.133 \\
\hline Anchoa lyolepsis & 2.228 & 0.037 & 3.846 & 0.133 \\
\hline Anchoa parva & 1.114 & 0.029 & 1.923 & 0.034 \\
\hline Anchoa sp & 0.836 & 0.018 & 1.442 & 0.019 \\
\hline Anchovia clupeoides & 0.557 & 0.038 & 0.962 & 0.009 \\
\hline Centengraulis edentulus* & 19.499 & 0.362 & 33.654 & 10.213 \\
\hline Lycengraulis grossidens & 1.114 & 0.015 & 1.923 & 0.033 \\
\hline Engraulidae indet.* & 18.106 & 98.845 & 31.250 & 55.847 \\
\hline
\end{tabular}




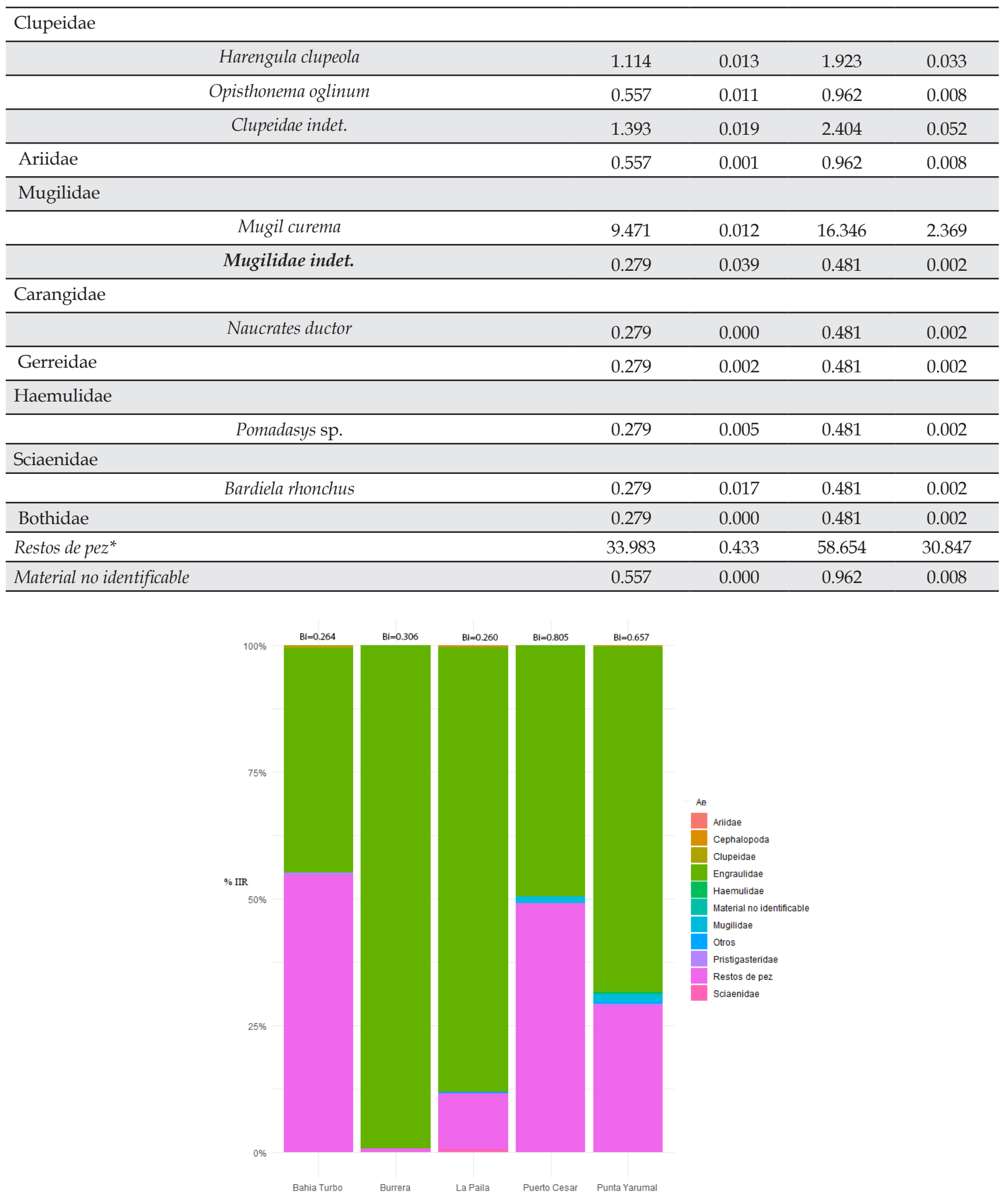

Figura 3. Índice de Importancia Relativa (\%IIR) de los ítems alimenticios en la dieta del pez Trichiurus lepturus en cada lugar de captura en el Golfo de Urabá, Caribe colombiano. 


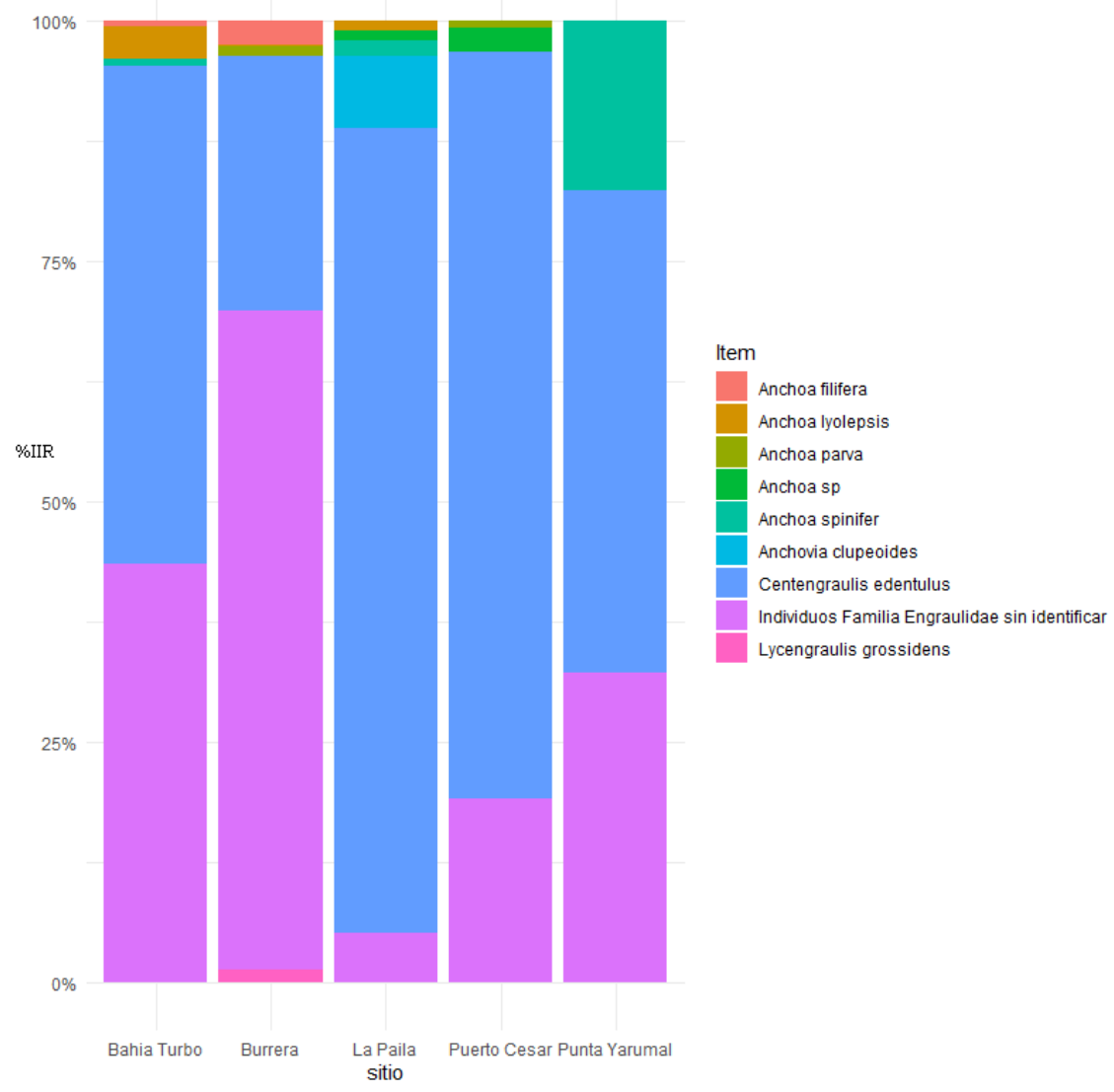

Figura 4. Índice de Importancia Relativa (\%IIR) de los ítems alimentarios pertenecientes a la familia Engraulidae en la dieta de los sables en cada lugar de captura en el Golfo de Urabá, Caribe colombiano.

La familia Engraulidae fue el principal componente alimentario en todos los lugares de muestreo, mientras que otras familias de peces, camarones y calamares fueron ocasionales en la dieta de los sables (Figura 3). Resaltó la presencia de Centengraulis edentulus y varias especies del género Anchoa (Figura 4). Sólo un 2.9\% de los peces analizados tenían presas recientes (estado 1), 17.8\% se encontraban en estados 2 (intermedio) y 3 (semidigerido), mientras que el $61 \%$ de los peces se encontraban en estado 4 (contenido digerido).

Se encontró traslape de la dieta de T. lepturus entre casi todos los lugares muestreados $(\mathrm{C} \lambda>0.6$; Tabla 2$)$, excepto entre Burrera y Puerto Cesar $(C \lambda=0.46)$. El no sobrelapamiento entre estos dos sitios se debió a que un estómago de T. lepturus capturado en Puerto Cesar en febrero de 2016 contenía 34 individuos juveniles del pez mugílido Mugil curema. Al calcular nuevamente el valor de solapamiento trófico excluyendo la presencia del Mugil curema, se obtiene $C \lambda=0.75$, valor que indica el solapamiento trófico en todos los sitios de muestreo. No se detectaron diferencias entre tallas, sexos y épocas climáticas.

No se encontraron diferencias en la dieta entre individuos talla pequeña (91 individuos) y grande (117 individuos); entre individuos de diferentes sexos (40 machos y 160 hembras) y entre capturados en las épocas climáticas (150 en época lluviosa y 58 en época seca) $(\mathrm{p}>0.05)$. 
Tabla 2. Resultados de solapamiento trófico del pez Trichiurus lepturus, entre sitios de captura en el golfo de Uraba, Caribe colombiano. Los resultados de sitios en los que no se traslapa la dieta están señalados con asterisco $\left(^{*}\right)$.

\begin{tabular}{|c|c|c|c|c|c|}
\hline & Puerto Cesar & Bahía Turbo & Burrera & La Paila & Punta Yarumal \\
\hline Puerto Cesar & & 0.73 & $* 0.46$ & 0.63 & 0.69 \\
\hline Bahía Turbo & & & 0.74 & 0.92 & 0.95 \\
\hline Burrera & & & & 0.90 & 0.88 \\
\hline La Paila & & & & & 0.99 \\
\hline Punta Yarumal & & & & & \\
\hline
\end{tabular}

\section{Discusión}

Los hábitos alimenticios del T. lepturus en el Golfo de Urabá involucran organismos similares a los reportados por Pardo et al. (2003), en la Bahía de Cartagena, Caribe colombiano, por Martins et al. (2005), Bittar et al. (2008) y Bittar et al. (2012) en aguas de Brasil, y en otras regiones (Wojciechowski, 1972; Zhang, 2004; Chiou et al., 2006; Yan et al., 2011; Rohit et al., 2015): en su mayoría peces, con aportes ocasionales de crustáceos y moluscos. Que los sables (piscívoros) se alimenten de organismos pelágicos, puede ser una estrategia para acortar el tiempo de búsqueda de alimento, minimizar energía en capturas y obtener mayor ganancia nutricional (Bittar et al., 2008; De la Cruz-Torres et al., 2014).

En esta investigación no se detectó variación estacional en la dieta de T. lepturus. Los engráulidos, representados por C. edentulus, se caracterizan por ser uno de los recursos pesqueros más abundantes durante todo el año en el Caribe colombiano, dada su baja comercialización (Coto et al., 1988) y sus elevadas tasas de crecimiento y fecundidad (Osorio \& Acero, 1996). En otras áreas, como Taiwán y Florida, se encontraron diferencias estacionales en la dieta, a causa de la alta actividad pesquera, que lleva a T. lepturus a consumir diferentes presas conforme a su disponibilidad a lo largo del año (Chiou et al., 2006; Bryan \& Gill, 2007).

Diversos autores han reportado canibalismo del T. lepturus (Wojciechowski, 1972; Nakamura \& Parin,
1993; Pardo et al., 2003; Martins et al., 2005; Chiou et al., 2006; De la Cruz-Torres et al., 2014), probablemente como resultado de una variación en la abundancia de presas a lo largo del año (Pájaro, 1998). Sin embargo, en este estudio no se encontró canibalismo, probablemente debido a la alta abundancia local de engráulidos, su presa más frecuente en el Golfo de Urabá (García \& Zaniboni, 2006). Esta abundancia, a su vez, puede ser por el efecto combinado de la posición tropical, la acción de los vientos, las corrientes marinas y el gran caudal del río Atrato (Ortiz \& Blanco, 2012), que proporcionan alta disponibilidad de nutrientes e impulsan la productividad biológica (Montoya, 2010), y a que la presencia de bosques de manglar favorece la abundancia de zooplancton (Giarrizzo et al., 2011) que es consumido por ellos. Sin embargo, Di Beneditto (2015) observó canibalismo en los sables a pesar de la elevada presencia de presas en el medio, pudiendo actuar este comportamiento como un mecanismo regulador de la densidad poblacional de la especie (Pájaro, 1998).

La distribución de los ítems en la dieta de los sables del Golfo de Urabá es diferente cuando se comparan las estaciones situadas en la boca del río Atrato con las estaciones de la costa oriental del Golfo (Figuras 1 y 3). Existe mayor frecuencia en el consumo de engráulidos en las áreas cercanas al delta del río (Burrera y La Paila), dónde se encuentran las mayores extensiones y desarrollo de los bosques de manglar (Ortiz \& Blanco, 2012) y disminuye a medida que se muestrea más lejos del río (Punta Yarumal, Bahía Turbo, Puerto Cesar). 
Por otro lado, existe una relación inversa entre la presencia de restos de peces y la proximidad al río Atrato: en los sitios cercanos al río disminuyen el índice de vacuidad y el porcentaje de digestión, lo cual soporta la conclusión de que los sables se alimentan con mayor frecuencia en zonas de manglar.

Los manglares son ecosistemas que ofrecen múltiples beneficios a peces asociados, sirviendo como zonas de refugio, áreas de crianza y de alimentación (Sandoval-Huerta et al., 2014), principalmente por la proliferación de copépodos (Laegdsgaard \& Johnson, 2001; Faunce \& Serafy, 2006; Nagelkerken et al., 2008). Al norte de Brasil, Giarrizzo et al. (2011) evidenciaron la importancia de áreas de manglar para la alimentación de distintas especies de engráulidos (Anchovia clupeoides, Anchoviella lepidontostole, Anchoa hepsetus), ya que son peces filtradores de fitoplancton y de zooplancton (Froese \& Pauly, 2019), con gran capacidad para utilizar eficazmente la oferta de recursos tróficos (González, 1981; Osorio \& Acero, 1996).

Debido a esto, se observa que en los sitios más cercanos a grandes coberturas de manglar (Burrera y La Paila) hay mayor diversidad en el consumo de especies de la familia Engraulidae (Figura 4), mientras que en Punta Yarumal, Bahía Turbo y Puerto Cesar, con menor cobertura de manglar, aumenta el consumo de otras familias de peces.

La alta incidencia de estómagos vacíos (índice de vacuidad $=35 \%$ ) puede ser consecuencia de una expulsión involuntaria durante su lucha por escapar de la red (Rohit et al., 2015). En los peces piscívoros el fenómeno de la regurgitación es común (Bakhoum, 2007), y está asociado con su estrategia de defensa y escape (Pérez et al., 2001; Reuben et al., 1997; Abdussamad et al., 2006; Ghosh et al., 2014; Yan et al., 2012).

Aunque el índice de repleción indicó que la mayoría de los sables (89.9\%) se encontraban en la categoría lleno, un alto número de estómagos presentaba eleva- dos porcentajes de digestión. Los peces carnívoros digieren rápidamente los contenidos estomacales, pues poseen un intestino corto y estómagos que segregan fuertes ácidos para digerir carne, huesos y escamas de las presas (Lagler et al., 1984; Rotta, 2003). Se recomienda no exponer por largos periodos de tiempo los individuos en las redes, para evitar que el pez digiera el alimento al momento de su captura, lo cual también afecta el coeficiente de vacuidad y la identificación de las presas.

Los cambios ontogénicos en los hábitos alimenticios de los sables son el resultado del aumento del tamaño del cuerpo, la boca y los dientes del pez a lo largo de su desarrollo, lo que le permite capturar una gama más amplia de tamaños y tipos de presas (Yan et al., 2012). Aunque otros estudios han encontrando variación en familias y especies consumidas a lo largo del desarrollo (Chiou et al., 2006; Yan et al., 2011), en esta investigación no se observaron estos cambios, ya que solo se obtuvieron dos grupos de tallas y ambos superan la talla media de madurez sexual, $(46.3 \mathrm{~cm}$; Froese \& Pauly, 2019), es decir que todos los individuos capturados son subadultos y adultos pequeños, según la clasificación realizada por Martins et al. (2005), y conservan el mismo rango de especies en el consumo trófico, aunque en diferentes proporciones.

La alta abundancia de Mugil curema en un único estomago lo cataloga como una presa circunstancial o accidental ( $\%$ IIR $=2.369 \%)$ en la dieta del sable. Esta especie de la familia Mugilidae, se caracteriza por ser pelágica en su estadío juvenil y consumir fitoplancton y zooplancton (Gámez et al., 2014), presas muy comunes en las áreas donde T. lepturus se alimenta.

En la actualidad gran cantidad de pesquerías están siendo sobreexplotadas (Robles, 2014; Riveiro, 2015; FAO, 2016), disminuyendo los grandes recursos pesqueros y aumentado la captura de peces de menores niveles tróficos (Pauly et al., 1998). Lawton \& Brown (1993) proponen que con la pérdida o d esequilibrio de los depredadores tope se reduce la estabilidad de las poblaciones de mesodepredadores 
(López, 2011), en las cuales se ubica T. lepturus. Los estudios centrados en especies del nivel trófico medio son una herramienta para medir la salud de los ecosistemas oceánicos, y para conocer la transferencia de energía y prevenir la pesca hacia la parte baja de la cadena trófica.

\section{Agradecimientos}

Los autores expresan su agradecimiento a los pescadores y al equipo de trabajo del Proyecto "Lineamientos Prioritarios para la Formulación de un Ordenamiento Pesquero del Golfo de Urabá" LOPEGU, del cual hizo parte esta investigación, dentro del Convenio especial de cooperación para la investigación no. 4600000983 celebrado entre el departamento de Antioquia - Secretaría de Agricultura y Desarrollo Rural y la Universidad de Antioquia, financiado por dichas entidades y el Fondo de Ciencia, Tecnología e Innovación del Sistema General de Regalías. Expresamos también gratitud al Grupo de Investigación en Sistemas Marinos y Costeros-GISMAC, a la Corporación Académica Ambiental y al equipo de trabajo de la Universidad de Antioquia Seccional Urabá, Colombia. Agradecemos la colaboración de Fernando José Parra Velandia por sus sugerencias. La recolección de especímenes de $T$. lepturus está amparada por el permiso marco No. 0524 de 27 de mayo de 2014 otorgado por la Autoridad Nacional de Licencias Ambientales (ANLA) y por el permiso No. 00001827 de 15 de octubre de 2015 de la Autoridad Nacional de Acuicultura y Pesca (AUNAP).

\section{Referencias}

Abdussamad, E. M., Nair, P. N. \& Achayya, P. (2006). The ribbonfish fishery and stock assessment of Trichiurus lepturus Linnaeus off Kakinada, east coast of India. Journal of the Marine Biological Association of India, 48(1), 41-45.

Agudelo, E., Ajiaco, R. E., Alvarez, L. E., Barreto, C. G., Borda, C. A., Bustamante, C. C., Caldas, J. P., De la
Hoz, J., Diazgranados, M. C., Melo, G., Perucho, E., Puentes, V., Ramírez, A., Ramírez, A., Rueda, M., Salinas, J. C. \& Zapata, L.A. (2011). Protocolo de captura de información pesquera, biológica y socio-económica en Colombia. Bogotá: Ministerio de Agricultura y Desarrollo Rural - Dirección de Pesca y Acuicultura - Subgerencia de Pesca y Acuicultura INCODER Conservación Internacional. 80 pp.

Al-Nahdi, A., Al-Marzouqi, A., Al-Rasadi, E. \& Groeneveld, J. C. (2009). The size composition, reproductive biology, age and growth of largehead cutlassfish Trichiurus lepturus Linnaeus from the Arabian Sea coast of Oman. Indian Journal of Fisheries, 56(2), 73-9.

Bakhoum, S. A. (2007). Diet overlap of immigrant narrowbarred Spanish mackerel Scomberomorus commerson (Lac., 1802) and the largehead hairtail ribbonfish Trichiurus lepturus (L., 1758) in the Egyptian Mediterranean coast. Animal Biodiversity and Conservation, 30(2), 147160.

Bax, N. J. (1998). The significance and prediction of predation in marine fisheries. ICES Journal of Marine Science, 55(6), 997-1030. https://doi.org/10.1006/jmsc.1998.0350

Bernal Franco, G., Toro Botero, F. M., Montoya Jaramillo, L. J. \& Garizábal, C. (2005). Estudio de la dispersión de sedimentos del río Atrato y sus impactos sobre la problemática ambiental costera del Golfo de Urabá. (Informe final de investigación). Medellín, Colombia: Universidad Nacional de Colombia. 61pp.

Bittar, V. T. \& Di Beneditto, A. P. M. (2009). Diet and potential feeding overlap between Trichiurus lepturus (Osteichthyes: Perciformes) and Pontoporia blainvillei (Mammalia: Cetacea) in northern Rio de Janeiro, Brazil. Zoologia, 26(2), 374-378. https://doi.org/10.1590/S1984-46702009000200023

Bittar, V. T., Castello, B. D. F. L. \& Di Beneditto, A. P. M. (2008). Hábito alimentar do peixe-espada adulto, Trichiurus lepturus, na costa norte do Rio de Janeiro, sudeste do Brasil. Biotemas, 21(2), 83-90. https:// doi.org/10.5007/2175-7925.2008v21n2p83

Bittar, V. T., Awabdi, D. R., Tonini, W. C. T., Vidal Junior, M. V., Beneditto, D. \& Madeira, A. P. (2012). Feeding preference of adult females of ribbonfish Trichiurus lepturus through prey proximate-com- 
position and caloric values. Neotropical Ichthyology, 10(1), 197-203.

https://doi.org/10.1590/S1679-62252012000100019

Blanco-Libreros, J. F. (2016). Cambios globales en los manglares del golfo de Urabá (Colombia): entre la cambiante línea costera y la frontera agropecuaria en expansión. Actualidades Biológicas, 38(104), 53. https://doi.org/10.17533/udea.acbi.v38n104a06

Blanco-Libreros, J. F., Ortiz-Acevedo, L. F. \& Urrego, L. E. (2015). Reservorios de biomasa aérea y de carbono en los manglares del golfo de Urabá (Caribe colombiano). Actualidades Biológicas, 37(103), 131-141. https://doi.org/10.17533/udea.acbi.v37n103a02

Borski, R. J. \& Hodson, R. G. (2003). Fish research and the institutional animal care and use committee. Ilar Journal, 44(4), 286-294. https://doi.org/10.1093/ilar.44.4.286

Bowen, S. H. (1996). Quantitative description of the diet. Fisheries techniques. 2nd edition. Bethesda, USA: American fisheries society. 513-532pp.

Brusca, R. \& Brusca, G. (2005). Invertebrados. México: McGraw-Hill Interamericana. 1005 pp.

Bryan, D. R. \& Gill, S. M. (2007). Seasonal occurrence of Atlantic cutlassfish, Trichiurus lepturus, in southeastern Florida with notes on reproduction and stomach contents. Florida Scientist, 70(3), 297-301.

Carpenter, K. E. \& De Angelis, N. (Eds.). (2002). The living marine resources of the Western Central Atlantic. Norfolk, Virginia, USA: Food and agriculture organization of the United Nations.

Cervigón, F., Cipriani, R., Fischer, W., Garibaldi, L., Hendrickx, M., Lemus, A. J., ... \& Rodríguez, B. (1992). Fichas FAO de identificación de especies para los fines pesca: Guía de campo de las especies comerciales marinas y de aguas salobres de la costa septentrional de Sur América. Roma, Italia: FAO.513 pp.

Chakraborty, A., Aranishi, F. \& Iwatsuki, Y. (2005). Molecular identification of hairtail species (Pisces: Trichiuridae) based on PCR-RFLP analysis of the mitochondral $16 \mathrm{~S}$ rRNA gene. Journal of applied genetics, 46(4), 381.

Chevillot, P., Molina, A., Giraldo, L. \& Molina, C. (1993). Estudio geológico e hidrológico del Golfo de Urabá. Boletín científico $\mathrm{CIOH}, 14,79-89$. https:/ / doi.org/10.26640/22159045.62

Chiappa, X., Gallardo, M. \& Jacob, M. (1989). Análisis del régimen alimentario de tres poblaciones de la anchoveta Engraulis mordax Girard (Pisces: Engraulidae), en el norte de Baja California. Anales del Instituto de Ciencias del Mar y Limnología . Universidad. Nacional Autónoma de México, 16(2), 361-378.

Chiou, W. D., Chen, C. Y., Wang, C. M. \& Chen, C. T. (2006). Food and feeding habits of ribbonfish Trichiurus lepturus in coastal waters of south-western Taiwan. Fisheries Science, 72(2), 373-381. https://doi.org/10.1111/j.1444-2906.2006.01159.x

Correa, I. D., Angel, R. \& Ded, J. (2002). Geología y oceanografía del delta del Río San Juan: litoral pacífico colombiano (No. 551.46 GEO). Medellín, Colombia: Fondo Editorial Universidad EAFIT.

Correa, I. D., Prüssmann, J. \& Garrido, A. E. (2010). Geomorfología del contorno litoral Urabá-Darién (Departamentos de Antioquia y Chocó, Caribe colombiano). En: Blanco, J.F., Londoño, M.H., Quan-Young, L., Urrego, L., Polanía, J., Osorio, A., Bernal, G. \& Correa, I. Expedición Estuarina, Golfo de Urabá. Comisión Colombiana del Océano. Fase, 1. 304 pp.

Coto, C. F., Luna, A. O., Calvo, A. L. \& Garcia, F. Z. (1988). Abundancia de algunas especies de Anchoas en la laguna de Términos (México), estimada a través de la captura de huevos. Annal del Institute de Ciência del Mar y Limnologia da Universidad Autónoma de México, 15(1), 125-134.

De la Cruz-Torres, J., Martínez-Pérez, J. A., Franco-López, J., \& Ramírez-Villalobos, A. J. (2014). Biological and ecological aspects of Trichiurus lepturus Linnaeus, 1758 (Perciformes: Trichiuridae) in Boca del Rio, Veracruz, Mexico. American journal of environmental sciences, 14, 1058-1066.

Di Beneditto, A. P. M. (2015). What drives the cannibalism of Trichiurus lepturus (Linnaeus, 1758) in the coastal area of southeastern Brazil (21-22 S)? International Journal of Fisheries and Aquatic Studies, 2(5), 363-365.

Díaz Murillo, B. P. (2010). Otolitos sagitta de especies selectas de Gerreidae (Teleostei: perciformes): variación interespecífica y su aplicación taxonómica. (Tesis Maestría en Ciencias). La Paz: CICIMAR-IPN. 82 pp. 
FAO. (2016). El estado mundial de la pesca y la acuicultura. Contribución a la seguridad alimentaria y la nutrición para todos. Roma: FAO.

Ferry, L. A., Clark, S. L. \& Cailliet, G. M. (1997). Food habits of spotted sand bass (Paralabrax maculatofasciatus, Serranidae) from Bahia de Los Angeles, Baja California. Bulletin of the Southern California Academy of Sciences, 96(1), 1-21.

Faunce, C. H. \& Serafy, J. E. (2006). Mangroves as fish habitat: 50 years of field studies. Marine Ecology Progress Series, 318, 1-18. https://doi.org/10.3354/meps318001

Franco, L. \& Bashirullah, K. M. B. (1992). Alimentación de la lisa (Mugil curena) del Golfo de Cariaco, estado Sucre, Venezuela; [The feeding of Mugil curena in the Cariaco Gulf, Sucre state, Venezuela]. Zootecnia Tropical, 10(2), 219-238.

Froese, R. \& Pauly, D. (2019). Editors FishBase. World Wide Web electronic publication. www.fishbase.org, version (04/2019).

Galván-Magaña, F., Nienhuis, H. J. \& Klimley, A. P. (1989). Seasonal abundance and feeding habits of sharks of the lower Gulf of California, Mexico. California Fish and Game, 75(2), 74-84.

Gámez Barrera, D., Morón Granados, E. \& Fuentes Reines, J. (2014). Description of the feeding habits of twelve species of fishes from the Ciénaga Grande de Santa Marta, Colombia. Boletin de Investigaciones Marinas y Costeras-INVEMAR, 43(1), 23-42.

García, C. B. (2010). Conocimiento tradicional: lo que los pescadores artesanales del Caribe colombiano tienen para decirnos. Pan-American Journal of Aquatic Sciences, 5(1), 78-90.

García, M., Pérez, J., López, T. \& Rodríguez, J. (2004). Descripción morfológica de los otolitos de las familias Engraulidae, Haemulidae y Achiridae del sistema estuarino de Tecolutla, Veracruz. Revista de Zoología, 15, 7-13.

García, V. J. A. \& Zaniboni Filho, E. (2006). El canibalismo en la larvicultura de peces. Revista MVZ Córdoba, 11(2), 9-19. https://doi.org/10.21897/rmvz.442

Ghosh, S., Rao, M. V., Rohit, P., Rammohan, K. \& Maheswarudu, G. (2014). Reproductive biology, trophodynamics and stock structure of ribbonfish Trichiurus lepturus from northern Arabian Sea and northern Bay of Bengal. Indian Journal of Geo-Marine Sciences, 43(5), 755-771.

Giarrizzo, T., Schwamborn, R. \& Saint-Paul, U. (2011). Utilization of carbon sources in a northern Brazilian mangrove ecosystem. Estuarine, Coastal and Shelf Science, 95(4), 447-457.

https://doi.org/10.1016/j.ecss.2011.10.018

González, E. (1981). Estudio bioecológico de los estadios juveniles de peces en la Ciénaga Grande de Santa Marta y zonas adyacentes. (Tesis de grado). Bogotá, Colombia: Universidad Nacional de Colombia. 89 pp.

Hobday, A. J., Bell, J. D., Cook, T. R., Gasalla, M. A. \& Weng, K. C. (2015). Reconciling conflicts in pelagic fisheries under climate change. Deep Sea Research Part II: Topical Studies in Oceanography, 113, 291-300. https:/ / doi.org/10.1016/j.dsr2.2014.10.024

Horn, H. S. (1966). Measurement of" overlap" in comparative ecological studies. The American Naturalist, 100(914), 419-424. https://doi.org/10.1086/282436

Hynes, H. B. N. (1950). The food of fresh-water sticklebacks (Gasterosteus aculeatus and Pygosteus pungitius), with a review of methods used in studies of the food of fishes. The Journal of Animal Ecology, 19(1), 36-58. https://doi.org/10.2307/1570

Hyslop, E. J. (1980). Stomach contents analysis - a review of methods and their application. Journal of fish biology, 17(4), 411-429. https://doi.org/10.1111/j.1095-8649.1980.tb02775.x Jobling, M. \& Breiby, A. (1986). The use and abuse of fish otoliths in studies of feeding habits of marine piscivores. Sarsia, 71(3-4), 265-274. https:// doi.org/10.1080/00364827.1986.10419696

Kapoor, B. G., Smit, H. \& Verighina, I. A. (1976). The alimentary canal and digestion in teleosts. Advances in marine biology, 13, 109-239.

https:/ / doi.org/10.1016/S0065-2881(08)60281-3

Krebs, C. (1989). Ecological Methodology. New York, USA: Harper and Row Publishers, 654 pp .

Laegdsgaard, P. \& Johnson, C. (2001). Why do juvenile fish utilise mangrove habitats? Journal of experimental marine biology and ecology, 257(2), 229-253.

https:/ / doi.org/10.1016/S0022-0981(00)00331-2

Lagler, K.F., Bardach, J., Miller, R. \& MayPassino, D. (1984). Ictiología. México: AGT. 489 pp. 
Lawton, J. H. \& Brown, V. K. (1993). Redundancy in ecosystems. En: Schulze ED., Mooney H.A. (eds) Biodiversity and Ecosystem Function. Praktische Zahnmedizin Odonto-Stomatologie Pratique Practical Dental Medicine (Geology), vol 99. Berlin, Alemania: Springer,. 255270 pp. https://doi.org/10.1007/978-3-642-58001-7_12

Leal-Flórez, J. (2017). Lineamientos Prioritarios para la Formulación de un Ordenamiento Pesquero del Golfo de Urabá - LOPEGU. (Informe Técnico). Turbo, Colombia: Universidad de Antioquia - Gobernación de Antioquia.

Levins, R. (1968). Evolution in changing environments: some theoretical explorations (No. 2). Princeton, USA: Princeton University Press. 132 pp.

López, A. (2011). Alteraciones de las redes tróficas marinas por efectos de pesca. Instituto Politécnico Nacional. (Tesis de Doctorado). La Paz, México: Centro Interdisciplinario de Ciencias Marinas. 55 pp.

Mann, H. B. \& Whitney, D. R. (1947). On a test of whether one of two random variables is stochastically larger than the other. The annals of mathematical statistics, 18 (1), 50-60. https://doi.org/10.1214/aoms/1177730491

Marrero, C. (1994). Métodos para cuantificar contenidos estomacales en peces. Caracas, Venezuela: Biocentro. $37 \mathrm{pp}$.

Martínez, J. A., Arteaga, M. M. C., Musi, J. L. T. \& Aranda, A. A. M. (2007). Utilización de otolitos como herramienta en la determinación de especies. Revista de Zoología, 18, 13-18.

Martins, A., Haimovici, M. \& Palacios, R. (2005). Diet and feeding of the cutlassfish Trichiurus lepturus in the Subtropical Convergence Ecosystem of southern Brazil. Marine Biological Association of the United Kingdom. Journal of the Marine Biological Association of the United Kingdom, 85(5), 1223. https://doi.org/10.1017/S002531540501235X

Merchant, P. F., Martínez, P. A., Chávez, A. M. \& Tello, M. J. L. (2008). Estructuras que se toman en cuenta para la descripción de los otolitos. Revista de Zoología, 19, 22-24.

Montoya Jaramillo, L. J. (2010). Dinámica oceanográfica del golfo de Urabá y su relación con los patrones de dispersión de contaminantes y sedimentos. (Doctoral dissertation). Medellín, Colombia: Universidad Nacional de Colombia. 254 pp.
Montoya, J. \& Toro, M. (2006). Calibración de un modelo hidrodinámico para el estudio de los patrones de circulación en el Golfo de Urabá. Revista Avances en Recursos Hidráulicos, 13,37-54.

Moreno, F., Acevedo, K., Grijalba-Bendeck, M., Polo-Silva, C. \& Acero, A. P. (2009). Espectro trófico de la raya eléctrica Narcine bancroftii (Griffith \& Smith 1834)(Elasmobranchii, Narcinidae) en playa Salguero, Santa Marta, Caribe Colombiano. Pan-American Journal of Aquatic Sciences, 4(4), 413-422.

Morzaria-Luna, H. N., Castillo-López, A., Danemann, G. D. \& Turk-Boyer, P. (2014). Conservation strategies for coastal wetlands in the Gulf of California, Mexico. Wetlands ecology and management, 22(3), 267-288. https://doi.org/10.1007/s11273-013-9328-0

Nagelkerken, I., Blaber, S., Bouillon, S., Green, P., Haywood, M., Kirton, L., Meynecke, J., Pawlik, J., Penrose, H., Sasekumar, A. \& Somerfield, P. (2008). The habitat function of mangroves for terrestrial and marine fauna: a review. Aquatic botany, 89(2), 155-185. https://doi.org/10.1016/j.aquabot.2007.12.007

Nakamura, I. (1993). Snake mackerels and cutlassfishes of the world (Families Gempylidae and Trichiuridae): FAO Fish. Syno, 125, 30-32.

Nelson, J. ( 2006). Fishes of the world. Hoboken, USA: John Wiley y Sons. 601 pp.

Ortiz, L. F. \& Blanco, J. F. (2012). Distribución de los gasterópodos del manglar, Neritina virginea (Neritidae) y Littoraria angulifera (Littorinidae) en la Ecorregión Darién, Caribe colombiano. Revista de Biología Tropical, 60(1), 219-232. https:// doi.org/10.15517/rbt.v60i1.2755

Osorio, A. C. \& Acero, A. (1996). Reproducción de Anchovia clupeoides y Anchoa parva (Pisces: Engraulidae) en dos ciénagas del Caribe Colombiano. Revista de Biología Tropical, 44(2), 781-793.

Pájaro, M. (1998). El canibalismo como mecanismo regulador denso-dependiente de mortalidad natural en la anchoíta argentina Engraulis anchoita. Su relación con las estrategias reproductivas de la especie. (Tesis de doctorado). Mar del plata, Argentina: Facultad de Ciencias Exactas y Naturales, Universidad Nacional del Mar del Plata. 187 pp. Pardo, F., Ospina, J. \& Álvarez, R. (2003). Hábitos alimenticios de algunas especies ícticas de la bahía de Cartagena 
y aguas adyacentes, Colombia. Dahlia-Revista Asociación Colombiana lctiólogos 6, 69-78.

Pauly, D., Christensen, V., Dalsgaard, J., Froese, R. \& Torres, F. (1998). Fishing down marine food webs. Science, 279(5352), 860-863. https:// doi.org/10.1126/science.279.5352.860

Pérez, L., Ajiaco, R. \& Ramírez, H. (2001). Aspectos biológicos de algunas especies de peces de interés ornamental en la baja Orinoquía colombiana. En: Ramírez-Gil H, Ajiaco Martínez RE. (eds.). La pesca en la baja Orinoquía colombiana: una visión integral. Bogotá: Instituto Nacional de Pesca y Acuicultura-INPA. 155- 210 pp.

Pinkas, L., Oliphant, M. S. \& Iverson, I. L. (1970). Food Habits of Albacore, Bluefin Tuna, and Bonito In California Waters. Fish Bulletin, 152, 105 pp.

Popper, A. N. \& Lu, Z. (2000). Structure-function relationships in fish otolith organs. Fisheries research, 46(1-3), 15-25. https:/ / doi.org/10.1016/S0165-7836(00)00129-6

Randall, J. (1967). Food habits of reef fishes of the West Indies. Studies in Tropical Oceanography, 5, 665-847.

Restrepo, J. \& Correa, I. (2002). Geología y oceanografía del delta del río San Juan: litoral pacífico colombiano. Medellín, Colombia: Fondo Editorial Universidad EAFIT. 221 pp.

Reuben, S., Vijayakumaran, K., Achayya, P. \& Prabhakar, R. V. D. (1997). Biology and exploitation of Trichiurus lepturus Linnaeus from Visakhapatnam waters. Indian Journal of Fisheries, 44(2), 101-110.

Riveiro Domínguez, P. (2015). La ordenación pesquera como instrumento para la práctica de una pesca sostenible en países en desarrollo (Estudio de caso de Guinea Bissau). Santiago de Compostela, España: Universidade de Santiago de Compostela. 515 pp.

Robles, E. (2014). Bienestar social y áreas naturales protegidas: Un caso de estudio en la costa de Oaxaca, México. Estudios sociales (Hermosillo, Son.), 22(44), 120-144.

Rohit, P., Rajesh, K. M., Sampathkumar, G. \& Sahib, K. (2015). Food and feeding of the ribbonfish Trichiurus lepturus Linnaeus off Karnataka, south-west coast of India. Indian Journal of Fisheries, 62(1), 58-63. https://doi.org/10.21077/ijf.2016.63.4.60190-06

Ros, R. M. \& Pérez C., M. (1978). Contribución al conocimiento de la biología del pez sable, Trichiurus lepturus (Linné1758).
La Habana, Cuba: Centro de Investigaciones marinas. 33pp.

Rotta, M. (2003). Aspectos gerais da fisiología e estrutura do sistema digestivo dos peixes relacionados á piscicultura. Documentos, 53, 1-48.

Rubio Limonta, M. \& Silveira Coffigny, R. (2009). Bienestar de los animales acuáticos, con fines de control sanitario. REDVET. Revista Electrónica de Veterinaria, 10(8).

Ruppert, E. \& Barnes, R. (1996). Zoología de los Invertebrados. México: McGraw - Hill Interamericana. 1114 pp.

Sandoval-Huerta, E. R., Madrigal-Guridi, X., EscaleraVázquez, L. H., Medina-Nava, M. \& DomínguezDomínguez, O. (2014). Estructura de la comunidad de peces en cuatro estuarios del Pacífico mexicano central. Revista mexicana de biodiversidad, 85(4), 11841196. https://doi.org/10.7550/rmb.42105

Shannon, C. E. (1948). The Mathematical Theory of Communication. The Bell System Technical Journal, $27,379-423$

Vaslet, A., Bouchon-Navaro, Y., Harmelin-Vivien, M., Lepoint, G., Louis, M. \& Bouchon, C. (2015). Hábitos de alimentación de peces arrecifales asociados con manglares y pastos marinos en una laguna del Caribe: Un enfoque de isótopos estables. Ciencias marinas, 41(3), 217-232.

Volpedo, A. V. \& Echeverria, D. D. (1999). Catalogo y claves de otolitos para la identificación de peces del mar de Argentina. Buenos Aires, Argentina: Editorial Dunken.

Windell, J. (1971). Food analysis and rate of digestion: Methods for assessment of fish production in fresh waters. Oxford, UK: Blackwell Scientific Publications. 215-226 pp.

Wojciechowski, J. (1972). Observations on biology of cutlassfish Trichiurus lepturus L.(Trichiuridae) of Mauritania Shelf. Acta Ichthyologica et Piscatoria, 2(2), 67-75. https:// doi.org/10.3750/AIP1972.02.2.08

Yan, Y., Hou, G., Chen, J., Lu, H. \& Jin, X. (2011). Feeding ecology of hairtail Trichiurus margarites and largehead hairtail Trichiurus lepturus in the Beibu Gulf, the South China Sea. Chinese Journal of Oceanology and Limnology, 29(1), 174-183. https:// doi.org/10.1007/s00343-011-0004-z 
Yan, Y., Chen, J., Lu, H., Hou, G. \& Lai, J. (2012). Feeding habits and ontogenetic diet shifts of hairtail, Trichiurus margarites, in the Beibu Gulf of the south China Sea. Acta Ecologica Sinica, 32, 18-25. https://doi.org/10.1016/j.chnaes.2011.04.008

Yáñez-Arancibia, A., Lara-Domínguez, A. L., Aguirre-León, A., Díaz-Ruiz, S., Amezcua-Linares, F., Flores-Hernández, D. \& Chavance, P. (1985). Ecología de poblaciones de peces dominantes en estuarios tropicales: Factores ambientales que regulan las estrategias biológicas y la producción. En: A. Yáñez-Arancibia (Ed.). Fish community ecology in estuaries and coastal lagoons: Towards an ecosystem integration. Pp. 311-366. México: UNAM. Zhang, B. (2004). Feeding habits and ontogenetic diet shift of hairfail fish (Trichiurus lepturus) in east china sea and yellow sea. Marine Fisheries Research, 25(2), 6-12. 


\section{Sara Arenas-Uribe}

Universidad de Antioquia

Turbo, Colombia

sara.au93@gmail.com

https://orcid.org/0000-0001-5612-4641

Jenny Leal-Flórez

Universidad de Antioquia

Turbo, Colombia

jenny.leal@udea.edu.co

https://orcid.org/0000-0002-5429-3975

\section{Alejandro Sandoval}

Universidad de Antioquia

Turbo, Colombia

sandovalejo@gmail.com

https://orcid.org/0000-0002-5595-1677

\section{Andres Felipe Pérez Villa}

Universidad de Antioquia

Turbo, Colombia

pereza.af95@gmail.com

https://orcid.org/0000-0002-3229-5099

\section{Andres Felipe Hernández-Morales}

Universidad de Antioquia

Turbo, Colombia

andresfelipe.1993@hotmail.com

Recibido: 22 de febrero de 2019

Aceptado: 22 de agosto de 2019

Hábitos alimenticios del pez sable Trichiurus lepturus en el Golfo de Urabá, Caribe colombiano

Citación del artículo: Arenas-Uribe, S., Leal-Flórez, J., Sandoval, A., Pérez-Villa, A.F. \& Hernández-Morales, A.F. (2019). Hábitos alimenticios del pez sable Trichiurus lepturus en el Golfo de Urabá, Caribe colombiano. Biota Colombiana, 20(2), 59-75. DOI: 10.21068/c2019.v20n02a05. 